INPLASY

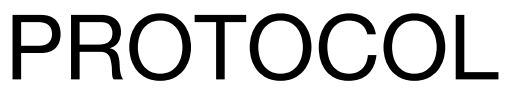

To cite: Song et al. Can sodium thiosulfate delay the progression of cardiovascular calcification in dialysis patients? a systematic review and meta-analysis. Inplasy protocol 202230018. doi: 10.37766/inplasy2022.3.0018

Received: 04 March 2022

Published: 04 March 2022

Corresponding author: Song Yuhuan

syuhuan@163.com

Author Affiliation:

Aerospace Center Hospital.

Support: Aerospace Center Hospital.

Review Stage at time of this submission: Data extraction.

Conflicts of interest:

None declared.

\section{Can sodium thiosulfate delay the progression of cardiovascular calcification in dialysis patients? a systematic review and meta-analysis}

Song, $\mathrm{YH}^{1}$; Cai, GY2; Peng, $\mathrm{T}^{3}$.

Review question / Objective: We performed a systematic review to synthesize the efficacy and safety of sodium thiosulfate (STS) in the progression of vascular calcification in dialysis patients.

Condition being studied: Over the years, up-to-date clinical studies targeting cellular mechanisms of calcification offer promising direction for drug development. At present, the available treatment choices for arterial calcification in dialysis include adequate dialytic therapy, use of non-calcium containing phosphate binders and calcimimetic agent, active vitamin D, myoinositol hexaphosphate, denosumab, tissuenonspecific alkaline phosphatase inhibitors and perhaps bisphosphonates and vitamin K. A promising approach is the use of STS, a chelating agent, which is commonly used for cyanide poisoning. In recent years, off-label treatment with STS is increasingly used for the treatment of soft tissue calcifications in calcific uremic arteriolopathy or calciphylaxis. Studies also found STS might delay the development of vascular calcification in dialysis indiviuals.

INPLASY registration number: This protocol was registered with the International Platform of Registered Systematic Review and Meta-Analysis Protocols (INPLASY) on 04 March 2022 and was last updated on 04 March 2022 (registration number INPLASY202230018).

\section{INTRODUCTION}

Review question / Objective: We performed a systematic review to synthesize the efficacy and safety of sodium thiosulfate (STS) in the progression of vascular calcification in dialysis patients.
Condition being studied: Over the years, up-to-date clinical studies targeting cellular mechanisms of calcification offer promising direction for drug development. At present, the available treatment choices for arterial calcification in dialysis include adequate dialytic therapy, use of non- 
calcium containing phosphate binders and calcimimetic agent, active vitamin $D$, myoinositol hexaphosphate, denosumab, tissue-nonspecific alkaline phosphatase inhibitors and perhaps bisphosphonates and vitamin $\mathrm{K}$. A promising approach is the use of STS, a chelating agent, which is commonly used for cyanide poisoning . In recent years, off-label treatment with STS is increasingly used for the treatment of soft tissue calcifications in calcific uremic arteriolopathy or calciphylaxis. Studies also found STS might delay the development of vascular calcification in dialysis indiviuals.

\section{METHODS}

Search strategy: The following search themes was used in various combinations: sodium thiosulphate, sodium thiosulfate, sodium hydrosulfite, dialysis and vascular calcification.

Participant or population: Dialysis patients.

Intervention: Sodium thiosulfate.

\section{Comparator: Control group with dialysis.}

Study designs to be included: PubMed, the Cochrane Library, Embase, Web of sciences, Ovid and Chinese science and technology databases were searched up to December 11, 2021 for RCTs and cohort studies to synthesize the efficacy and safety of STS in the progression of vascular calcification in dialysis patients. Primary outcome was coronary artery calcification scores (CACS) or abdominal aortic calcification scores (AACS). Secondary outcomes were pulse wave velocity (PWV). Laboratory datas were shown in safety data.

Eligibility criteria: Over the years, up-todate clinical studies targeting cellular mechanisms of calcification offer promising direction for drug development. At present, the available treatment choices for arterial calcification in dialysis include adequate dialytic therapy, use of noncalcium containing phosphate binders and calcimimetic agent, active vitamin D, myoinositol hexaphosphate, denosumab, tissue-nonspecific alkaline phosphatase inhibitors and perhaps bisphosphonates and vitamin K. A promising approach is the use of STS, a chelating agent, which is commonly used for cyanide poisoning. In recent years, off-label treatment with STS is increasingly used for the treatment of soft tissue calcifications in calcific uremic arteriolopathy or calciphylaxis. Studies also found STS might delay the development of vascular calcification in dialysis indiviuals. Therefore, to guide clinical practice and bring light on vascular calcification, we performed the systematic review to evaluate the safety and efficacy of STS treatment on vascular calcification, as measured by coronary artery calcification score or abdominal aorta calcification score, the PWV and chronic kidney disease-mineral bone disease indexes.

Information sources: PubMed, the Cochrane Library, Embase, Web of sciences, Ovid and Chinese science and technology databases.

Main outcome(s): Seven RCTs and 7 cohort studies including 590 patients were included. Four RCTs reported a significant reduction in the changes of CACS or AACS in dialysis patients. Two RCTs showed a beneficial effect on PWV. Bicarbonate changes were observed in 3 RCT studies and in 3 cohort studies. Significant differences were not observed in the change of hsCRP and PTH. The most common adverse events were temporary nausea and vomiting in $12.5 \%$ to $75 \%$ of patient.

Quality assessment / Risk of bias analysis: Quality assessment of each study was independently analysed by YHS and GYC. We used the Cochrane Risk of Bias Tool for RCTs and the Newcastle-Ottawa Quality Assessment Scale (NOS) for observational researches.

Strategy of data synthesis: Statistical metaanalysis of the included studies was subjected by the Review Manager (Version 5). Due to the differences in evaluation methods and sections, we measured the 
standard mean difference (SMD) and 95\% Cls for continuous index. $P$ values less than 0.05 represented statistical significance. While there were established outcomes from two or more studies, meta-analysis was applied. Statistical heterogeneity besides trials was investigated operating the $I^{2}$ statistical trials and $p$ values. Values with $\mathrm{p}<0.1$ and $\mathrm{I}^{2}$ larger than $50 \%$ indicated the heterogeneity was significant. In view of the limited number of included studies or patients, the heterogeneity of statistical test may be insensitive. The random-effect model was adopted to integrate each result. We performed subgroup analysis according to the RCTs or cohort studies. We performed the leave-one-out method to evaluate the sensitivity and heterogeneity. We carried out the funnel plots to detect publication bias.

Subgroup analysis: We performed subgroup analysis according to the RCTs or cohort studies.

Sensitivity analysis: We performed the leave-one-out method to evaluate the sensitivity and heterogeneity.

Country(ies) involved: China.

Keywords: sodium thiosulfate; dialysis; vascular calcification.

Contributions of each author:

Author 1 - SONG Yuhuan.

Author 2 - CAl guangyan.

Author 3 - PENG Ting. 